\title{
Cognitive Model of Space Information Networks
}

\author{
Liu Hui
The PLA military representative \\ Liu Hui
The PLA military representative \\ office of 764 factory \\ Tianjin , China \\ e-mail: huaxuexue1990@163.com
}

\author{
Zheng Zhong \\ Communication Institute, \\ Equipment Academy of Air Force \\ Beijing, China \\ e-mail: zhzh_2008@qq.com
}

\author{
HuaXuexue \\ Communication and Information System \\ Northeastern University \\ Shenyang, China \\ e-mail: huaxuexue1990@163.com
}

\author{
ShiJiping \\ Dalian People's Liberation Army Air Force \\ Communications Officer Academy \\ Dalian, China \\ e-mail: shijiping@163.com
}

\begin{abstract}
With the characteristics of highly dynamic topology and limited-resources, space information network are difficult to match its huge applications requirements. These inherent characteristics make it vulnerable to security risks. Comparing to traditional terrestrial networks, it is more vulnerable to its environmental and security threats. At the same time, the space information network plays an important role in the national economy and military applications, it puts forward high demands on network security. Risk assessment can predict and diagnose the network security threats, provide decision-making basis for security policy and counter measures. It become an important part of network security protection system.To solve this problem, cognitive wireless network technology is used and the cognitive model of space information network is proposed. This model is based on annular cognitive structure, it consists of multi-domain sensing, intelligent decision and network reconfiguration. The network is divided into three layers, the local multi-domain cognitive layer, the regional collaborative cognitive layer and the network overall cognitive layer. Cross-layer method of the node is designed, thus the nodes can have the cognitive ability. Finally, it is to be achieved that the traditional module of passive adaptive environment can be changed and the space information networks can adapt to the surrounding environment.
\end{abstract}

Keywords- space information networks;cognitive technologies; multi-domain cognitive; risk assessment; cognitive model

\section{INTRODUCTION}

As a new network, Space information networks[1-2] is distributed, self-organizing and self-management. With the development of aerospace technology, business needs of the space networks towards diversification, individuation and broadband. With the characteristics of highly dynamic topology and limited-resources, space information network are difficult to match its huge applications requirements. In space information networks, how to use the limited resources effectively to meet the huge application requirements becomes the key problem urgently to be solved. In the existing space information network, it doesn't put forward effective solution to solve the problem between network resources and applications demand. In order to solve this problem, the network needs a full understanding of itself.

Network cognitive technology is inspired by the cognitive function of biological systems. On the one hand its cognitive characteristics are beneficial to realize the self-management and self-running. On the other hand, the cognition of internal and external network environment can complete the deep interaction of network. It can improve the performance of the network as a whole and the port to port system. It is the inevitable trend of the next generation communication network [3-9].

Currently, the network cognitive technology has received extensive attention from academia, business and military industry. They put forward a lot of typical network cognitive architectures. S.Shakkottai et al. [10] proposed cross-layer design and provide a solution to the network architecture design with a ability of cognition. In 2006, Motorola et al. [11] proposed a FOCALE cognitive network architecture. They introduce the autonomic computing into the cognitive network. By the context awareness and fuzzy recognition technology, it enables the system to adapt to users' needs dynamically and achieve the simplification of business management.. But these cognitive frame structure are geared to the specific areas.

In this paper, we propose a cognitive model of space information networks.It makes the space information networks have an accurate understanding of performance and resource usage. Besides, it can make us take a strategic approach to using network resources, and 
ultimately maximize the network resource utilization and network efficiency.

\section{COGNITIVE MODEL OF SPACE INFORMATION NETWORK}

The structure of space information network is large and complex and the nodes are heterogeneous. So it has brought challenges to its network management effectiveness. The cognitive technique of cognitive wireless network is a perfect network intelligent management solution. Based on the cognitive principle of cognitive wireless network, we design a cognition model for the space information network. It can collect, process, analysis, and report the network status accurately and real-time. So it can provide the foundation for network intelligent decision and guarantee for network

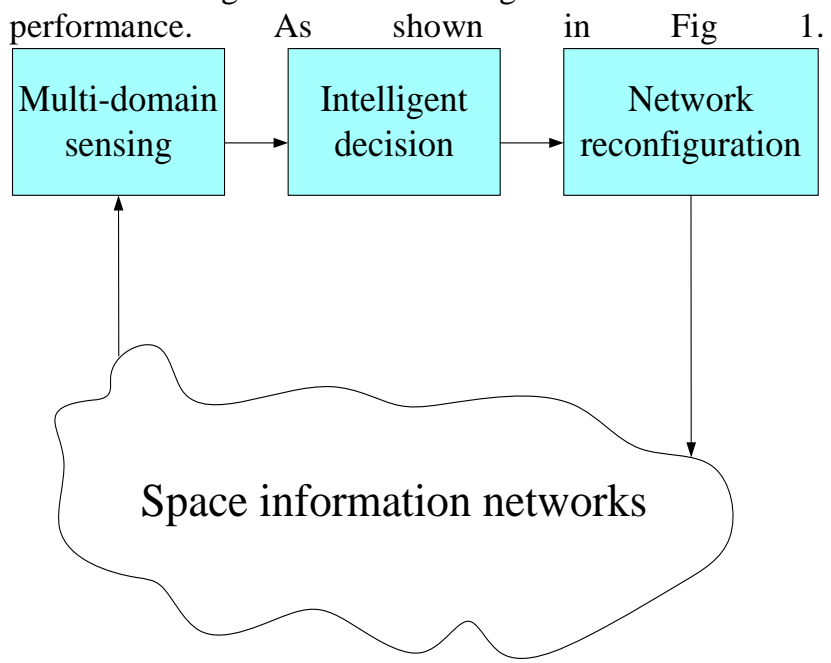

Figure 1. Cognitive structure of space information network

This module mainly consists of multi-domain sensing, intelligent decision and network reconfiguration. Multidomain sensing senses the information from environment and evaluates the current status of network. It also should submit the results to the intelligent decision. Then the intelligent decision module gives reconstitution decision to the network reconfiguration module. By the network reconstitute module is responsible for the execution of the decision, adjusting the network parameters appropriately and making the network self-adaptive to the environment.

\section{A. Multi-domain Sensing Module}

Because of the heterogeneity and high dynamic performance of space information network, it will cause the significant uncertainty of information. The distribution regularity of multi-domain environment [12] is described by parameters that can reflect its performance. Different environment domain has its unique and associated parameters. Multi-domain environment sensing module divides the internal and external environment of the network into space environment, network environment, and user environment. It collects and processes data in this three cognitive environment domains.

The parameter information of space environmental domain embodies the wireless resource utilization, such as link capacity, channel utilization and spectrum holes.
From domain parameter information of network environment we can get heterogeneous network type recognition, quality of routing, network performance and the configuration. User environment parameters are refers to some application layer parameters information such as the application of network type, user preferences and the QoS requirements of business. The users' need dynamically matches with the wireless network capacity because of the cognition of users' environment enables, so it ensures the network performance. Therefore, the relationship is close in three environmental domains. We must consider the multi-objective optimization to coordinate parameters and get intelligent control decisions.

Multi-domain environment sensing is the basis of the space information network status cognition. It observes the behavior of network, supervises the performance of network, makes directional decision, and judges whether the network needs adjustment. If the network is considered normal, it continues to run; If the network was found to be abnormal, it will give information to the intelligent decision module and find the best reconfiguration strategy.

\section{B. Intelligent Decision Module}

Intelligent decision module can achieve the function of self-organizing and self-management. When the network founds its performance can not be able to meet the business requirements, it will find the appropriate solution through the intelligent decision module. In the intelligent decision module, it needs to complete the function of reasoning, planning and learning. As shown in Fig 2. The input data is network state information provided by multi-domain environment sensing module. The output data is the reconfiguration scheme through case matching and algorithm study. The intelligence of space information network will be reflected on this. When meets unfamiliar cases it will use a reasoning algorithm that like the human brain thinking and give a multiobjective optimization scheme. If the cases have been reported, it will give a solution quickly.

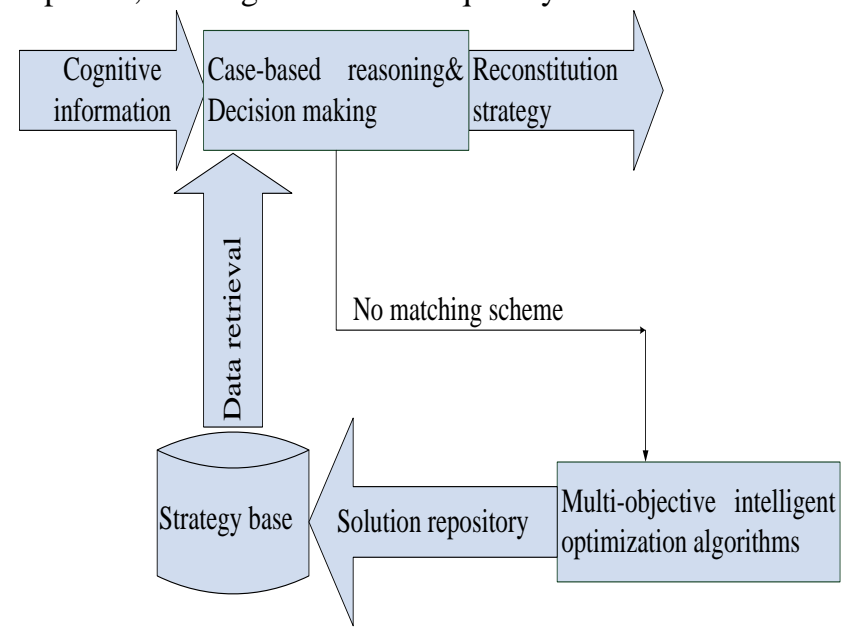

Figure 2. The processing of intelligent decision 


\section{Network Reconfiguration Module}

Network reconfiguration module is responsible for the self-healing function of the space information network. So it can guarantee the continuous operation of the entire network. Network reconfiguration of space information network can be divided into four levels: application, network, link and equipment. The more the network is damaged, the lower the reconfiguration level. Space information network reconfiguration process is shown in Fig.3.

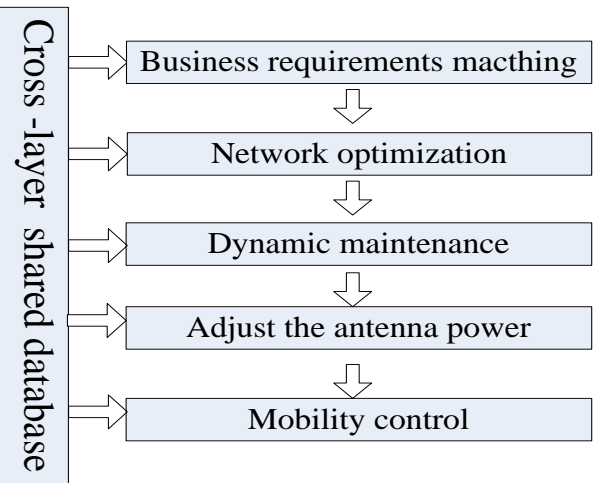

Figure 3. Space information network reconfiguration process

1) The application refactoring: It needs to take multiple objective QoS index optimization and QoS level negotiation to solve the problem when the business requirements beyond the network service ability.

2) The network refactoring: It includes routing and topology.

3) The link refactoring: It's usually caused by network link breaking down or node's move. It can implement the link level reconstruction of the network through the power control, node's move and routing algorithm.

4) The equipment refactoring: The equipment refactoring based on software radio technology. It will adjust the running parameters and behavior of equipment unit when the equipment malfunction, failure and no longer be trusted.

\section{Nodes DePloyment OF THE NETWORK COGNITION}

\section{A. The Classification of the Network Cognition}

The nodes deployment of space information network uses the hierarchical architecture, because the hierarchical architecture not only has efficient management and throughput, but also easy locates the nodes. It is necessary to adopt hierarchical architecture to manage the space information network.

The nodes deployment of the space information network is divided into three layers. The bottom is a multi-domain local cognitive layer, the second layer is the regional coordinative cognitive layer and the top layer is the overall cognitive layer. The design idea is: the top layer (network center nodes) is responsible for information management and control of the entire network. The middle layer (this layer can be divided into some rank) not only process its own regional information, but also finish the information relay to the senior center. The bottom local nodes as sensors are responsible for collecting vast amounts of information to classify and collate. In addition, it manages and controls the local state and uploads the useful information to the upper center nodes after the local information processing. In this way, the cognitive information can be spread in the network step by step. The scope is bigger and bigger, but only a small amount of useful information is in diffusion. So it greatly reduces the network congestion and burden. Network nodes deployment is shown in Fig 4. The network level cognition is divided into three levels: the bottom is multi-domain local cognitive layer, then the Regional collaborative cognitive layer and the top is the network overall cognitive layer

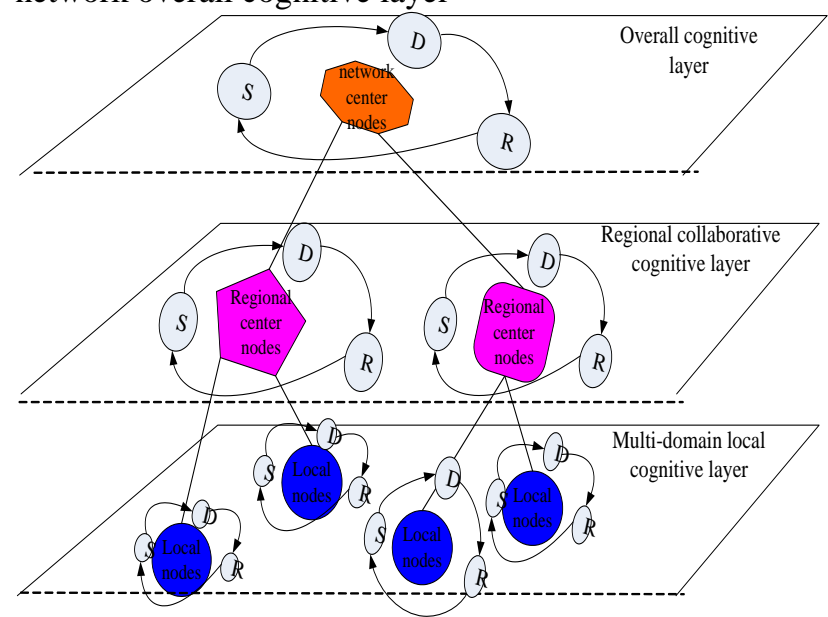

Figure 4. The cognitive levels of space information network

\section{B. The nodes cognitive process}

The space information network that has cognitive ability can be thought as a multi-agent network. In this paper we divide network cognition into there layers: the multi-domain local cognitive layer, the regional coordination cognitive layer and the overall cognitive layer. At the same time, in order to achieve networking process according to the specific business, the nodes can be divided into three categories: the center nodes, the regional center nodes and the local cognitive nodes. Network center nodes refer to a node or a few nodes launch a new business, namely the source nodes. In the database of the local cognitive nodes only store the local data. The local cognitive nodes are responsible for the formation of the local state diagram and adjusting timely. The local regional center nodes need collect the single point cognitive information uploaded by the local nodes, form regional state diagram and upload to the network center nodes.

After the completion of networking, cognitive nodes are required to get large amounts of cognitive information. In this article, cognitive nodes use a cross-layer design of the existing shared database. It will process the cognitive information in the cognitive layer, review the network status and extract the reconstruction scheme.

\section{TEST}

We use network security risk assessment to test the cognitive model. Regardless of whether the network security attacks, nodes can, according to the network 
security status and history of the health of its current network security risk assessment, and with the operation of the network, the resulting value is also changing the risk assessment. Network as a basis, automatically take accurate safety measures and counter-measures to ensure the safe operation of the network. The simulation part is to verify the safety risk assessment really happening when the attack did not take security measures and countermeasures. If the assessment is correct, the risk assessment resulting higher the value, the corresponding network performance deterioration more severe. The simulation results shown in Fig 5 and Fig 6.

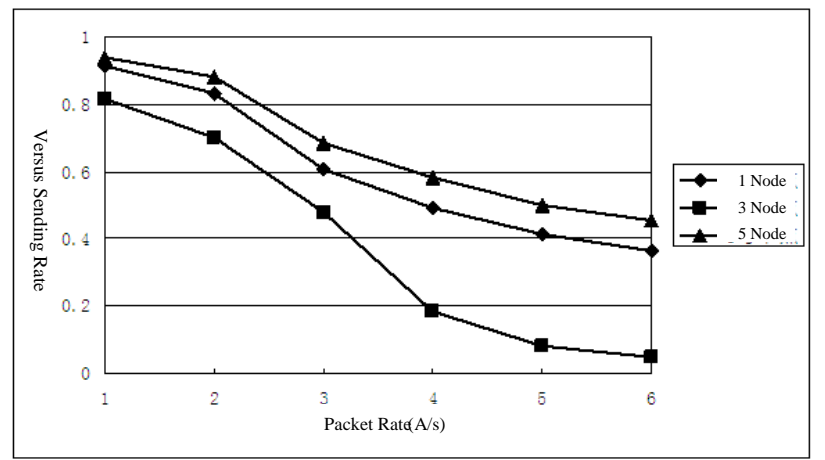

Figure 5. Throughput versus sending rate under three nodes

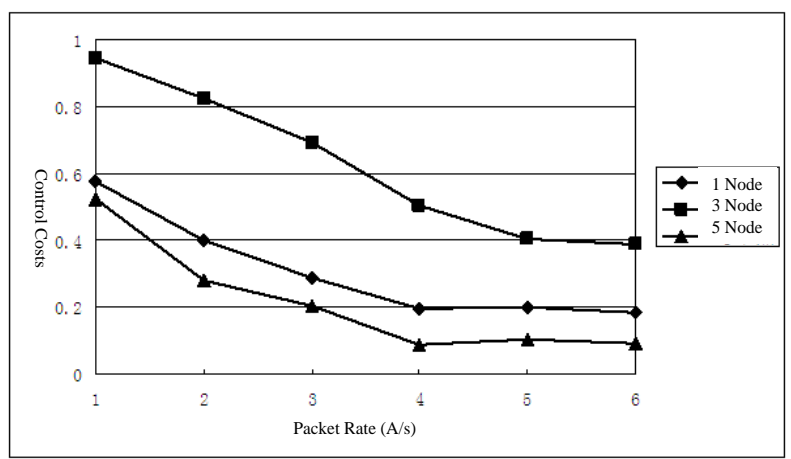

Figure 6. Overhead versus sending rate under three nodes

\section{CONCLUSION}

This article puts forward cognitive model of the space information network because the cognitive wireless network technology can sol ve the special application requirements and characteristics of the network. The space information network is designed as a new network with cognitive ability. It can turn the network from the traditional model of passive adaptive environment into a new model which study and comply with the environment. And we design the node deployment of the space information network in detail. In addition we get the cognitive process and cognitive architecture of the network. The three layers of the cognitive network are the multi-domain local cognitive layer, the regional coordination cognitive layer and the overall cognitive layer. At the same time nodes are divided into the center nodes, the regional nodes and the local cognitive nodes. We can use the three kinds of nodes together to complete network cognitive process.

\section{REFERENCES}

[1] Liujun. "Research on Security Networking Key Technologies of Space Information Networks" [D], Shenyang, Northeastern University, 2007.

[2] De Sanctis M., Rossi T., Lucente M., Ruggirei M., Mortari D. "Space system architectures for Interplanetary Internet" [C], Aerospace Conference, IEEE, Big Sky, MT March, 2010.

[3] W.Thomas. "Cognitive Networks"[D].Virginia Polytechnic Institute and State University,2007.

[4] Thomas R W,DaSiva L A,MacKenzie A B. "Cognitive Networks” [Z].New Frontiers in Dynamic Spectrum Access Networks.2005,pp.352-360.

[5] Liuchao, WangHaitao. "A New Trend in the Development of Network-Cognitive Network"[J], Data Communications, 2009, 2,pp.1-5.

[6] GongZhenghu, ZhuoYing. "Research on Cyberspace Situational Awareness"[J], Journal of Software,2010,27(7),pp.1605-1619

[7] GuChengjie, ZHangShunshun, SunYanfei. "QoS Self-Adaptive Control in Cognitive Networks Based on Service Awareness", ZTE Technology Journal, 2011,17(1),pp.49-52.

[8] MITOLA J. Cognitive radio: Making software radios more personal [J]. IEEE Personal Communications, 1999, 6(4),pp. 13-18.

[9] TRAPPE W. "Cognitive radio in US: overview, challenge and directions" [EB/OL], http://www.winlab.rutgers.edu,2007.

[10] Srivastava V, Motani. "Cross-layer Design: A Survey the Road Ahead" [J].IEEE Communication Magazine,2005,43(12),pp.112119.

[11] Strassner J,Agoulmine N,Lehtihet E.FOCALE. "A Novel Autonomic Computing Architecture"[C] //Proc. of Latin American Autonomic Computing Symposium. Campo Grande, MS, Brazil, July 2006.

[12] WangJinlong, WuQihui, GongYuping. "Cognitive wireless networks”[M]. Beijing: China Machine Press, 2010. 